The Astrophysical Journal, 268:689-697, 1983 May 15

() 1983. The American Astronomical Society. All rights reserved. Printed in U.S.A.

\title{
NOVA SHELLS
}

Judith G. Cohen AND ANn J. Rosenthal

Palomar Observatory, California Institute of Technology

Received 1982 August 20; accepted 1982 November 10

\begin{abstract}
A search to find spatially resolved nova shells was carried out; two new shells (around FH Ser 1970 and V533 Her 1963) were discovered. The angular sizes measured at the current epoch of the eight previously known shells around novae which erupted prior to 1945 imply that the shells are coasting out at constant velocity. Expansion velocities, interstellar absorptions, and masses of the ejected material were determined for several recent novae. A new calibration of the maximum visual luminosity-rate of decline relationship for galactic novae is given.
\end{abstract}

Subject headings: nebulae: general - stars: novae

\section{INTRODUCTION}

Classical novae at maximum light appear to obey a luminosity-rate of decline relationship, as summarized by McLaughlin (1960) and reviewed by van den Bergh (1975). This makes them in principle useful as an extragalactic distance indicator. The most reliable distance determinations for galactic novae are those obtained from the angular expansion of nova shells, of which nine are now known. In $\S$ II we present the results of a search for more such shells, during which two new resolved shells were discovered. Our spectroscopic observations of novae are presented in $\S$ III. These data give rise to a new calibration of the maximum luminosity-rate of decline relationship described in $\S$ IV.

\section{THE SEARCH FOR NOVA SHELLS}

The 60 inch $(1.5 \mathrm{~m})$ telescope of the Palomar Observatory was used with the digital SIT detector (Kent 1979) to photograph square fields $3^{\prime}$ on a side during the 1981 observing season. Photographs were taken through an interference filter centered at $6563 \AA$ with a full width at half-maximum transmission of $30 \AA$. One pixel corresponds to $00^{\prime \prime} 72$ at the focal plane. Novae which were at maximum light brighter than $V=5$ were photographed, generally with $1 \mathrm{hr}$ exposures. Because of instrumental problems, shells with radii smaller than $3^{\prime \prime}$ around stars brighter than $V=14$ cannot be detected.

Many of the previously known classical nova shells were observed to check on their current size and detectability. Radii of these shells (corrected for the pincushion distortion of the detector as determined from observation of a projected rectangular grid) are listed in Table 1, as are their expected radii based on the early observations compiled by McLaughlin. If the shells are noticeably elliptical, the semimajor axis is tabulated.
During the course of this survey, two new shells were found, around FH Ser 1970 and V533 Her 1963. These shells are barely resolved in the present data. The average of horizontal and vertical cuts through their images, as well as that of a nearby star on the same frame as each of the novae, are shown in Figure $1 .^{1}$ The profiles are shown as functions of the accumulated count in a pixel (normalized to the accumulated count of the central pixel) versus offset in pixels from the point of maximum brightness of the image. Note that because of the low dynamic range of the SIT detector, it was impossible to go below $1 / 100$ of the central brightness.

We first consider the projected spatial intensity profile of a point source plus a shell. It is assumed that the shell is always optically thin in the emission lines. The intrinsic profile depends somewhat on whether the total volume of the sphere of radius $R$ is emitting, or only a shell of inner radius $(1-a) R$ and outer radius $R$. Another parameter necessary to describe the intrinsic spatial intensity profile is the ratio of the brightness of the star to the brightness of the shell in the central pixel, denoted $I^{*} / I(\mathrm{sh})$. For a fixed ratio $I^{*} / I(\mathrm{sh})$, it is immediately apparent that a totally filled shell $(a=1)$ produces an intensity profile with a minimum amount of light beyond the spatial extent of the point source; therefore, this intrinsic profile will yield a maximum estimate of the radius of the shell. We therefore adopt $a=1$ and compute such profiles for a range of values of $I^{*} / I(\mathrm{sh})$ and $R$. These intrinsic profiles are then convolved with the point spread function as determined from a star image on the same frame as the nova. A

\footnotetext{
${ }^{1}$ The profiles shown for FH Ser 1970 in Figs. 1 and 2 are actually from a frame taken through the same $\mathrm{H} \alpha$ filter on the 60 inch telescope at Palomar Mountain in 1982 August, using a prototype direct CCD detector whose pixels are squares 0.47 on a side.
} 
TABLE 1

Sizes of Nova SHELlS

\begin{tabular}{|c|c|c|c|}
\hline \multirow[b]{2}{*}{ NAME } & \multicolumn{2}{|c|}{ RADIUS (arcsec) } & \multirow[b]{2}{*}{ NOTES } \\
\hline & Observed (1981) & Expected & \\
\hline V603 Aql ... & not detected & 60 & \\
\hline T Aur ......... & 9.5 & 10.5 & 1 \\
\hline V476 Cyg ... & 5.7 & 5.7 & \\
\hline DQ Her .... & 10.5 & 12.7 & 1 \\
\hline CP Lac ........ & not detected & 11.3 & \\
\hline GK Per...... & 41.5 & 43.2 & 1 \\
\hline CP Pup ....... & 7 & 8.2 & 2 \\
\hline RR Pic ....... & 11.5 & 9.7 & 1,3 \\
\hline HR Del...... & 1.8 & $\ldots$ & 1,4 \\
\hline FH Ser $\ldots \ldots$ & 2.0 & $\ldots$ & \\
\hline V533 Her ... & 1.6 & $\ldots$ & \\
\hline BT Mon .... & 3.8 & $\ldots$ & \\
\hline
\end{tabular}

Notes.-(1) Shell non-spherical. (2) Radius from Williams 1982. (3) Radius from Williams and Gallagher 1979. (4) Radius from Kohoutek 1981.

comparison of calculated profiles for filled shells with the spatial intensity profiles of the two novae gives a reasonable fit, as shown in Figure 2, for $R=1.6$ and $I^{*} / I(\mathrm{sh})=3$ for V533 Her and for $R=22^{\prime \prime 3}$ with $I^{*} / I(\mathrm{sh})=1$ for $\mathrm{FH}$ Ser. The profile of FH Ser can also be fit by a thin shell $(a=0.2)$ with $R=1^{\prime \prime} .9$ and a larger value of $I^{*} / I(\mathrm{sh})$ (although the total shell luminosity is approximately the same for both models). As a compromise we adopt $2{ }^{\prime \prime} 0$ as the radius for $\mathrm{FH}$ Ser. We note that in order for these shells to appear resolved at all, they must have radii of at least 0 .' 8 .
A check on the spatial modeling can be obtained by comparing the ratio of the stellar continuum flux over the $30 \AA$ filter bandpass to the emission line flux with the ratio $I^{*} / I(\mathrm{sh})$. From the spectra described in $\S$ III $d$, taken through an aperture larger than the shell, and using the radii of the shells derived above, we obtain $I^{*} / I$ (sh) (per arcsec squared) as 1.5 for $\mathrm{FH}$ Ser, while the weaker emission lines in V533 Her relative to the continuum imply $I^{*} / I$ (sh) (per arcsec squared) about 3 . This agrees well with that deduced from the best fits to the spatial profiles.

In addition, J. B. Oke and R. A. Wade (private communication) have discovered a shell around BT Mon 1939, a nova which unfortunately was discovered only in its late decline stage (Payne-Gaposchkin 1957).

\section{SPECTROSCOPIC OBSERVATIONS}

\section{a) Expansion Velocities}

The spectra of many novae have been observed over the past two years, mostly with the $5 \mathrm{~m}$ Hale telescope at Palomar Mountain. A few objects were observed with the $2.5 \mathrm{~m}$ du Pont telescope at the Las Campanas Observatory. For each object, observations using either the intensified reticon detector (Shectman and Hiltner 1976) or the CCD detectors on the Double Spectrograph (Oke and Gunn 1982a) were obtained at sufficiently high dispersion to resolve the emission lines. Modeling of the expected line profiles of shells as seen in spectra centered on the postnova star with apertures of various dimensions relative to the shell radius indicate that the

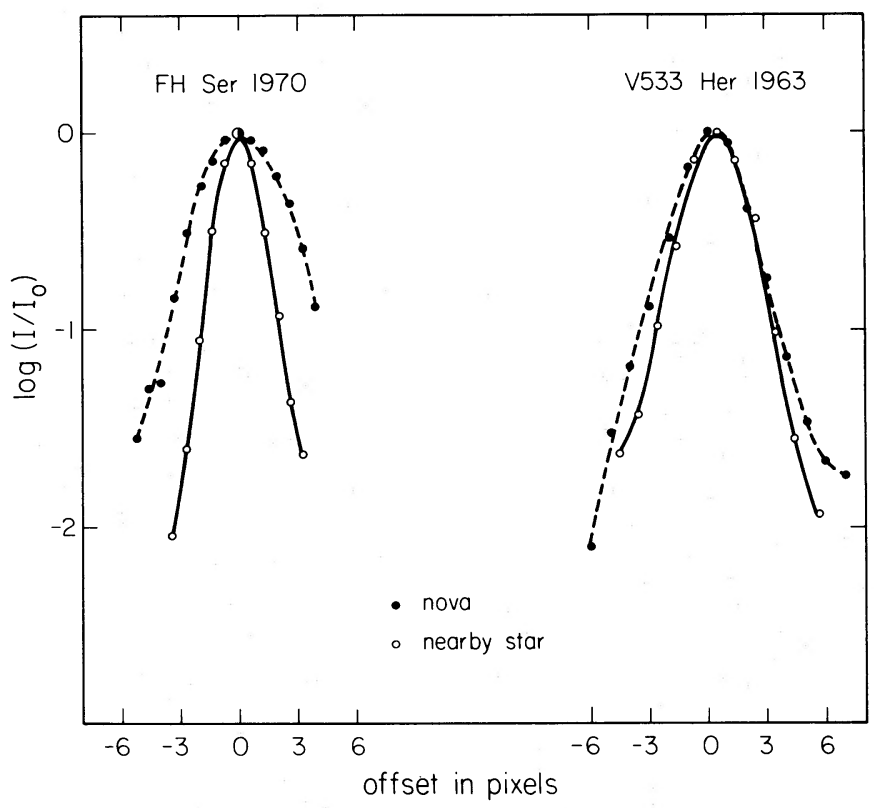

FIG. 1.-The spatial intensity profile through a narrow band H $\alpha$ filter of FH Ser 1970 and V533 Her 1963 (solid circles) as compared with nearby stars (open circles) of similar magnitude from the same direct SIT frames (see footnote 1). 


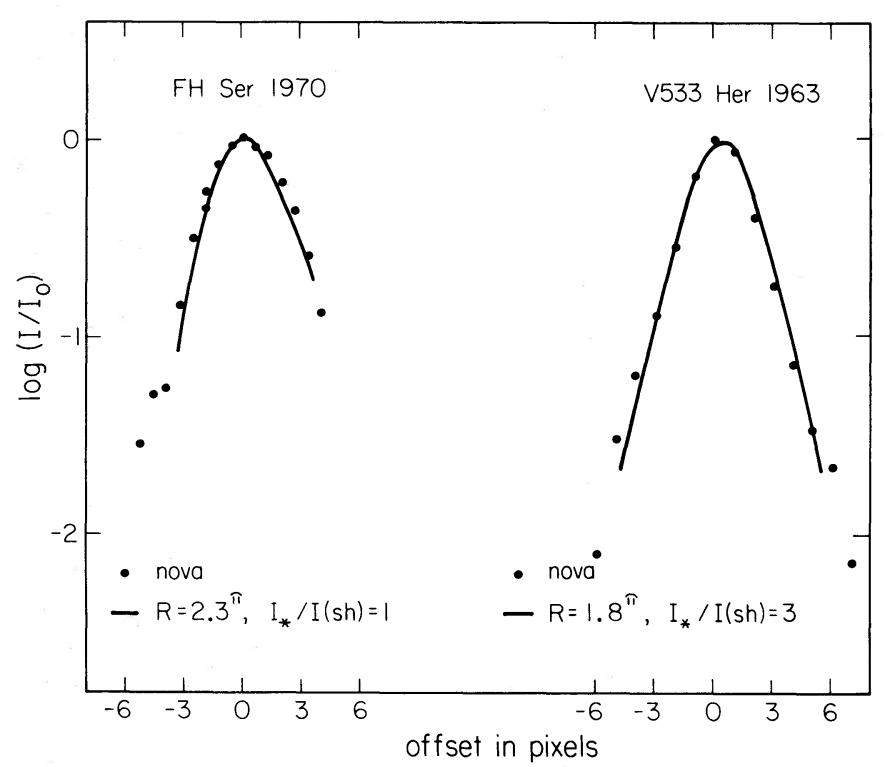

FIG. 2. - The spatial intensity profile through a narrow band H $\alpha$ filter of FH Ser 1970 and V533 Her 1963 (solid circles) as compared to profiles computed for a point source plus a shell (smooth curves).

full width at half-maximum of the emission lines is a suitable measure of twice the expansion velocity of the shell. Generally $\mathrm{H} \beta, \mathrm{H} \gamma$, and $4686 \mathrm{He}$ I were used.

In Table 2 we list the expansion velocities and the resolution ( 3 pixels) for the novae we have observed. For the objects observed with a CCD detector, the resolution (followed by an asterisk in Table 2) is that of 1 pixel. For the few novae observed only at the lowest resolution, the somewhat more uncertain values of $v(\exp )$ are denoted by colons. Except for these cases, the values of $v(\exp )$ are accurate to $\pm 50 \mathrm{~km} \mathrm{~s}^{-1}$. The velocities determined for CP Lac and V603 Aql must refer to the accretion disc, as no shell was detected photographically. The $v$ (exp) compiled by McLaughlin for these two novae are given in parentheses following the values we measured.

The shells of GK Per and DQ Her are much larger than the $2^{\prime \prime} \times 2^{\prime \prime}$ entrance aperture we used with the intensified Reticon detector. Photographs of these shells show many discrete condensations, and thus our spectra could underestimate the expansion velocity if by chance there was no emission knot within the small aperture we used either in the front or rear portion of the shell. The $v(\exp )$ for these two novae are therefore adopted from Humason (1943) and from McLaughlin, respectively. For the remaining four novae listed in McLaughlin's compilation in which the shell is still detectable, the mean difference between our measurements and those tabulated by him is $+90 \mathrm{~km} \mathrm{~s}^{-1}$, with a dispersion about the mean of $78 \mathrm{~km} \mathrm{~s}^{-1}$. Thus nova shells are coasting out at constant velocity, at least over a time scale reaching up to 90 years. This is not surprising, as deceleration is expected only once the shell has swept up a significant fraction of its own mass in interstellar material, which for typical nova shells expanding into a medium of density 1 atom $\mathrm{cm}^{-3}$, requires about 500 years.

\section{b) New Identifications of Novae}

We note that the identification suggested by Klemola (1968) for V368 Aq1 1936 is spectroscopically confirmed. Star 4 on Klemola's chart has emission in the hydrogen lines and 4686. Some absorption lines are probably also present, particularly the $3933 \AA$ line of Ca II. Also Humason's (1938) probable identification of Nova Sgr 1898 is confirmed. The star has the Balmer lines and 4686 in emission.

The very large $v$ (exp) found for Nova CrA 1981 indicates that this object is of particular interest. Its spectrum, as observed on 1981 May 20 and 25, shows a number of unusual strong emission lines at $6830 \AA$ (Ca X?) and $4720 \AA$ (Ne IV?).

Identifications for Nova Sct 1970, Nova Aql 1970, and Nova Vul 1976 are shown in Figure 3. High scale photographs are essential to locate these objects now that they have faded below 15 th mag.

\section{c) Interstellar Lines and Reddening}

These spectra can also be used to search for interstellar lines and hence determine the absorption to these objects. The interstellar lines detected, excluding upper limits, are listed in Table 3, while the interstellar absorption values deduced using the ratios of $N(\mathrm{Na} \mathrm{I}) / A_{V}$ and 
TABLE 2

Expansion Velocities, Reddenings, and Light Curve Parameters

\begin{tabular}{|c|c|c|c|c|c|c|c|}
\hline Object & $\begin{array}{c}V_{\exp } \\
\left(\mathrm{km} \mathrm{s}^{-1}\right)\end{array}$ & $\begin{array}{l}\text { Resolution } \\
\text { (A) }\end{array}$ & $\begin{array}{c}A_{V} \\
(\mathrm{mag})\end{array}$ & $\begin{array}{l}V(\max ) \\
(\operatorname{mag})\end{array}$ & $\begin{array}{c}t_{2} \\
\text { (days) }\end{array}$ & $M_{V}$ & Notes \\
\hline \multicolumn{8}{|c|}{ Known Shells } \\
\hline V603 Aql.. & $265(1700)$ & 1.2 & 0.2 & -1.1 & 4 & -9.15 & \\
\hline T Aur ....... & 655 & 1.2 & 1.5 & 4.2 & 80 & -7.9 & \\
\hline V476 Cyg...... & 790: & $8.1^{*}$ & 0.6 & 2.0 & 7 & -9.85 & \\
\hline HR Del $\ldots \ldots \ldots \ldots$ & 520 & 1.2 & 0.2 & 4.8 & $>150$ & -5.05 & 1 \\
\hline DQ Her $\ldots \ldots \ldots \ldots$ & 315 & $<1.0$ & 0.2 & 1.4 & 67 & -6.2 & 2 \\
\hline V533 Her............ & 580 & $2.2^{*}$ & 0.2 & 3.5 & 26 & -7.45 & 3 \\
\hline CP Lac ............ & 295: $(1600)$ & $8.1^{*}$ & 0.8 & 2.1 & 5 & -9.35 & \\
\hline BT Mon $\ldots \ldots \ldots \ldots$ & 800 & 1.2 & & $\ldots$ & $\ldots$ & & 4 \\
\hline GK Per $\ldots \ldots \ldots \ldots$ & 1200 & 1.2 & 0.3 & 0.2 & 6 & -8.55 & 5 \\
\hline RR Pic $\ldots \ldots \ldots \ldots$ & 475 & 1.7 & 0.2 & 1.2 & 80 & -7.3 & \\
\hline CP Pup ............ & 710 & 1.7 & 0.3 & 0.4 & 5 & -9.55 & \\
\hline FH Ser $\ldots \ldots \ldots \ldots$ & 560 & 1.2 & 2.8 & 4.4 & 42 & -7.55 & 6,7 \\
\hline \multicolumn{8}{|c|}{ Recent Novae } \\
\hline Aql $1970 \ldots \ldots \ldots$ & 575 & 1.7 & $\ldots$ & $\ldots$ & $\ldots$ & $\cdots$ & \\
\hline CrA $1981 \ldots \ldots \ldots \ldots$ & 2210 & 3.6 & $\ldots$ & $\ldots$ & $\cdots$ & $\ldots$ & \\
\hline V1500 Cyg $1975 \ldots$ & 1180 & 1.2 & 1.1 & 1.85 & 2.4 & -9.45 & 8 \\
\hline Cyg $1978 \ldots \ldots \ldots \ldots$ & 735 & 1.2 & $\ldots$ & $\ldots$ & $\ldots$ & $\ldots$ & \\
\hline Per $1974 \ldots$. & 630 & 1.2 & $\ldots$ & $\ldots$ & $\ldots$ & $\ldots$ & \\
\hline Sct $1975 \ldots \ldots \ldots \ldots$ & 840 & 3.6 & $\ldots$ & $\ldots$ & $\ldots$ & $\ldots$ & \\
\hline CT Ser $1948 \ldots \ldots$. & 535 & 1.2 & $\ldots$ & $\ldots$ & $\ldots$ & $\ldots$ & \\
\hline Ser $1978 \ldots \ldots \ldots \ldots$ & 600 & $2.2^{*}$ & 1.0 & 7.9 & 36 & -4.95 & 9,10 \\
\hline Sgr $1978 \ldots \ldots \ldots \ldots$ & 785 & 3.6 & $\ldots$ & $\ldots$ & $\ldots$ & $\ldots$ & \\
\hline Sgr $1980 \ldots \ldots \ldots \ldots$ & 825 & 3.6 & $\cdots$ & $\cdots$ & $\ldots$ & $\ldots$ & \\
\hline Vul $1968 \ldots \ldots$ & 455 & 1.2 & $\ldots$ & $\ldots$ & $\ldots$ & $\ldots$ & \\
\hline NQ Vul 1976. & 705 & $2.2^{*}$ & 1.4 & 6.0 & 18 & -6.05 & 10,11 \\
\hline
\end{tabular}

NotES. - (1) Light curve from Barnes and Evans 1970. (2) $V_{\exp }$ from Humason 1943. (3) Light curve from Chincarini 1964. (4) Discovered long after maximum light. (5) $V_{\exp }$ from McLaughlin 1960. (6) $A_{V}$ adopted from Gallagher and Code 1974. (7) Light curve from Borra and Anderson 1970. (8) Light curve from Young et al. 1976. (9) Light curve from AAVSO as given in Gehrz et al. $1980 a$. (10) $M_{V}$ rejected; see text. (11) Light curve from AAVSO Circular No. 76 (1977).

$N\left(C_{\text {II }}\right) / A_{V}$ inferred by Cohen (1975) are listed in the fourth column of Table 2. For the older novae included in McLaughlin's review, the light curve parameters and the absorption are taken from McLaughlin's tables, with the exception of $\mathrm{T}$ Aur. That object's interstellar lines lead to a discrepant absorption of $1.5 \mathrm{mag}$, as compared to the tabulated value of $0.7 \mathrm{mag}$. Gallagher et al. (1980) deduced $A_{V}$ approximately $1.9 \mathrm{mag}$ from the $\mathrm{H} \alpha / \mathrm{H} \beta$ Balmer line ratio. It is thus clear that in this case the absorption has been substantially underestimated in the past.

An apparent distance modulus- $E(B-V)$ curve was constructed for 14 stars of spectral type A5 and earlier within $1^{\circ}$ of NQ Vul 1976 from the photometric catalog of Blanco et al. (1970). From this curve, using an approximate distance of $1400 \mathrm{pc}$ (see $\S$ IV), we obtain $A_{v}=1.4 \mathrm{mag}$.

\section{d) Masses of the Shells}

For those shells smaller in diameter than $10^{\prime \prime}$, we obtained spectrophotometric scans through a $10^{\prime \prime}$ diam- eter aperture centered on the post-nova star using the Double Spectrograph (see Oke and Gunn 1982a) on the $5 \mathrm{~m}$ telescope of Palomar Observatory. During each of two photometric nights in 1982 June several standard stars from Oke and Gunn (1982b) were observed for flux calibration. Figure 4 shows the spectrum of FH Ser 1970 observed with the CCD detectors on the Double Spectrograph. We note that the spectrum of Nova Cyg 1920 appears to show evidence of an $\mathrm{M}$ star contribution in that the TiO band at $7600 \AA$ is apparently weakly present.

In the first part of Table 4 the absolute fluxes (not corrected for interstellar absorption) measured from these spectra are listed for the stronger emission lines with definite identifications. The second part of the table contains the remaining definitely present emission features (excluding several Balmer and Paschen lines) as well as the emission lines in the region $5100-5700 \AA$. This interval is poorly covered in our spectrophotometric scans due to the cutoff of the dichroic used in the Double Spectrograph; other spectra taken without the 

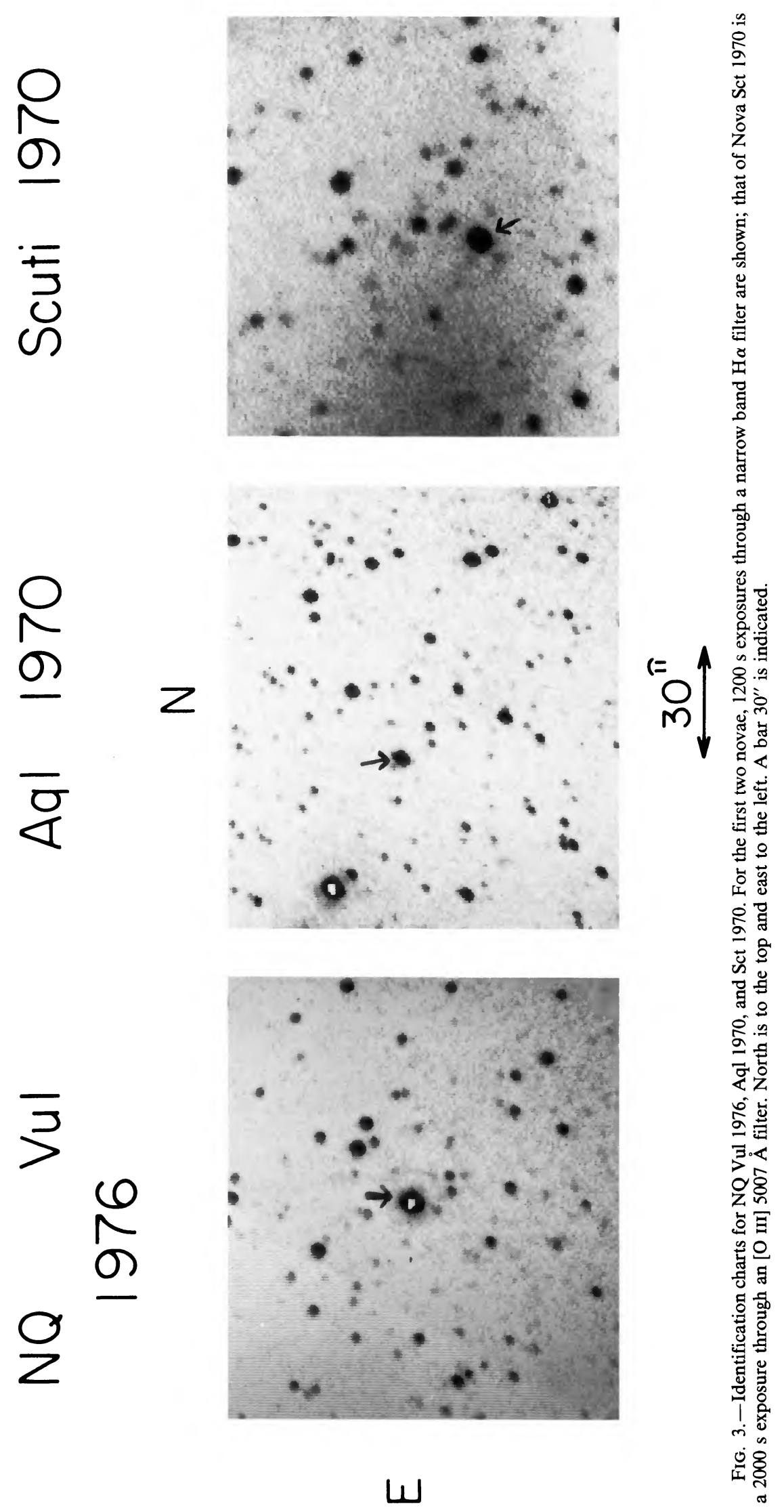
TABLE 3

INTERSTELLAR LINES

\begin{tabular}{cccc}
\hline \hline Object & $\begin{array}{c}\text { Na I }\left(\mathrm{D}_{1}+\mathrm{D}_{2}\right) \\
W_{\lambda}(\AA)\end{array}$ & $\begin{array}{c}\text { Ca II (3933) } \\
W_{\lambda}(\AA)\end{array}$ & $\begin{array}{c}\text { Ca II (3968) } \\
W_{\lambda}(\AA)\end{array}$ \\
\hline T Aur ............ & $\ldots$ & 1.1 & 0.9 \\
HR Del ........ & 0.4 & $\ldots$ & $\ldots$ \\
V533 Her $1963 \ldots$ & 0.4 & $\ldots$ & $\ldots$ \\
\hline
\end{tabular}

dichroic, often through a slit $2^{\prime \prime}$ wide, were used to fill in this gap, but absolute fluxes cannot be obtained for these lines. Those definitely present are indicated as $\mathbf{P}$, while the more dubious ones are indicated as P.: The wavelengths of the features are given in the first column. In the cases where the wavelength of a particular feature in a given nova seems to be slightly different, the last two digits are given in parentheses following the absolute flux. The final row of Table 4 lists the absolute flux in the continuum at $6000 \AA$ for each object.

We assume an electron temperature of $10,000 \mathrm{~K}$, a shell thickness $a$ of $10 \%$ of the shell radius, and a filling factor $b$ within this shell of $10 \%$ and use the $\mathrm{H} \beta$ absolute fluxes corrected for interstellar absorption to derive the electron densities and shell masses of hydrogen only listed in Table 5. Given the uncertain but large $\mathrm{He}, \mathrm{C}$, $\mathrm{N}$, and $\mathrm{O}$ abundances (see, for example, Williams 1982), the total shell masses may be up to a factor of 2 larger than the tabulated values. Note that the derived $n_{e}$ is relatively insensitive to the choice of $T_{e}$ within a reasonable range ${ }^{2}$, but $n_{e} \propto(a b)^{-1 / 2}$ and mass $\propto(a b)^{1 / 2}$. As the shell expands and evolves, both these parameters decrease with time to approximately the values used above, which are from our unpublished measurements of old resolved shells. It is more difficult to determine these parameters for the youngest shells. Thus the rather low shell masses derived for some of the more recent novae in Table 5 are undoubtedly due to underestimates of $a$ and $b$. When the same geometrical factors are used, our mass for the shell of HR Del agrees to within a factor of 2 with that derived by Robbins and Sanyal (1978) from the $\mathrm{H} \beta$ flux observed in 1969.

Given the complexities of the analysis of the spectra of nova shells, reviewed by Williams (1977), we have decided not to attempt at present to derive abundances from our emission-line fluxes but rather to leave this for a future publication. It is clear, however, that FH Ser 1970 has a very high carbon abundance.

\footnotetext{
${ }^{2}$ If the shells are extremely cool, as is the case for DQ Her 1934, where the electron temperature now is about $500 \mathrm{~K}$ (Williams et al. 1978), the shell masses and electron densities listed in Table 5 are too large by a factor of 3 .
}

\section{THE ABSOLUTE MAGNITUDE-RATE OF DECLINE RELATIONSHIP}

Distances using the kinematic expansion of spatially resolved nova shells are now available for 11 novae using the data of Tables 1 and 2. For the two novae whose shells have faded below the limit of detectability, we use McLaughlin's compilation for their shell size. The visual magnitudes at maximum light and the time in days to decline $2 \mathrm{mag}\left(t_{2}\right)$ of the eight classical shells are also taken from that source. Recorded novae in the direction of globular clusters were reviewed by Hogg (1973). T Sco 1860 probably is a member of the globular cluster M80, whose distance and reddening we adopt from Harris and Racine (1979). Its light curve is reviewed by Payne-Gaposhkin (1957).

An extensive series of photometric observations from the visible to the infrared of V1500 Cyg (Gallagher and Ney 1976), NQ Vul (Ney and Hatfield 1978), and Nova Ser 1978 (Gehrz et al. 1980a) have identified time intervals during which each of these novae apparently were radiating like blackbodies. Combining values of $A_{V}$ with the observed flux distribution of the nova as a function of wavelength yields an effective temperature and an angular diameter of the radiating blackbody at each appropriate time. This, together with $v(\exp )$ and the data of the outburst, determines a distance. The distances for these three novae were derived using the values of $A_{V}$ and $v$ (exp) of Table 2. Note that the $V$ magnitude at maximum light for Ser 1978 is that advocated by Gehrz et al. (1980a) as an extrapolation of the first two observations (separated by 4 days). The interstellar absorption for this nova is not well determined. Gehrz et al. $1980 a$ adopt $A_{V}=1.0 \mathrm{mag}$. The ratio of the Balmer line strengths in Table 4 indicates that $A_{V}$ cannot be larger than this value. We also adopt 1.0 mag for Nova Ser 1978. The absorption to Nova Cyg 1975 is taken from the reddening-distance map of Young et al. (1976) and the distance determined using the infrared photometry of Gallagher and Ney (1976) with our expansion velocity. There is a slight inconsistency in that Gallagher and Ney do not correct their observed energy distribution for interstellar absorption, but beyond $1 \mu \mathrm{m}$ the corrections are in any case small. A similar inconsistency occurs in the $A_{V}$ used to correct the infrared observations of Nova Vul 1976 (1.8 mag) versus the 1.4 mag adopted here. Distances inferred from such photometry are somewhat suspect as they depend on the chosen $A_{V}$ and on the assumption that the nova is optically thick and radiates like a blackbody.

The resulting absolute visual magnitudes of the 14 novae are listed in the penultimate column of Table 2, except for T Sco with $M_{v}=-9.4$. The absolute visual magnitude-rate of decline relationship is given in Figure 5. Nova Cyg 1975 lies close to the mean relationship, while the other two novae with distances determined 


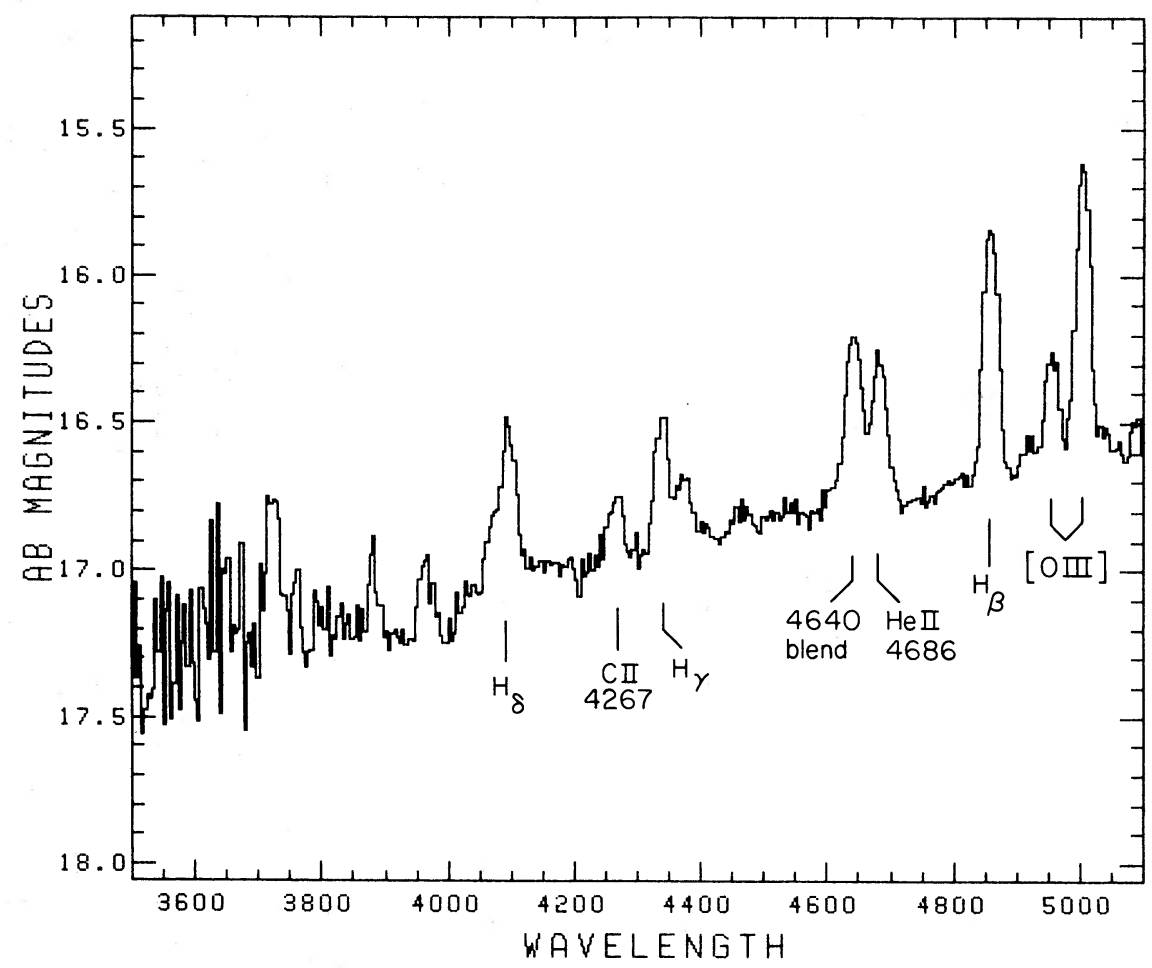

FIG. $4 a$

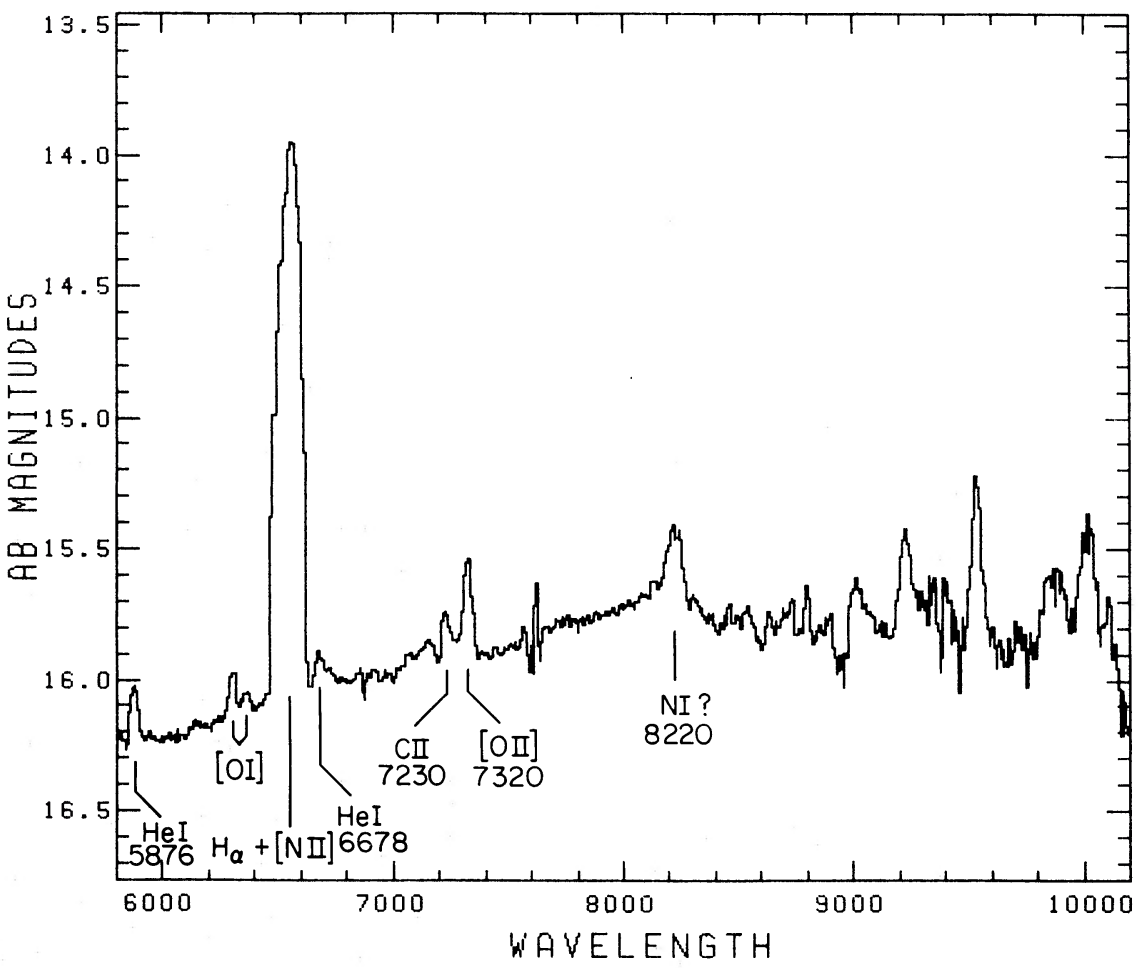

FIG. $4 b$

FIG. 4.- Spectrophotometric scans from the Double Spectrograph (Fig. $4 a$ is the blue camera spectrum, while Fig. $4 b$ is from the red camera) of FH Ser 1970 are shown. The exposure was $1800 \mathrm{~s}$ through a $10^{\prime \prime}$ entrance aperture. 
TABLE 4

OBSERVED ABSOLUTE FluXES

\begin{tabular}{|c|c|c|c|c|c|c|c|c|}
\hline Line & Ser 1978 & $\begin{array}{c}\text { NQ Vul } \\
1976\end{array}$ & $\begin{array}{c}\text { V1500 Cyg } \\
1975\end{array}$ & Aql 1970 & $\begin{array}{c}\text { FH Ser } \\
1970\end{array}$ & $\begin{array}{c}\text { HR Del } \\
1967\end{array}$ & $\begin{array}{c}\text { V533 Her } \\
1963\end{array}$ & Cyg 1920 \\
\hline \multicolumn{9}{|c|}{ Strong Emission Lines (units: $10^{-15} \mathrm{ergs} \mathrm{s}^{-1} \mathrm{~cm}^{-2}$ ) } \\
\hline $\mathrm{Pa} 7(9545)+[\mathrm{S} \mathrm{IIII}] \ldots$ & $\ldots$ & 8.0 & 3.1 & $\ldots$ & 7.5 & 95 & 3.8 & $\ldots$ \\
\hline$[\mathrm{O}$ II $] 7320 \ldots \ldots \ldots \ldots$ & $\ldots$ & 5.0 & $\ldots$ & $\ldots$ & 7.3 & 31 & & \\
\hline He I $6678 \ldots$ & $\ldots$ & 1.4 & $\ldots$ & $\ldots$ & 2.2 & 13 & 3.9 & 1.4 \\
\hline [N II] 6584 & 51 & ) & ] & 46 & 225 & 1400 & & \\
\hline $\mathrm{H} \alpha 6562 \ldots$ & 18 & 380 & 110 & 10 & 60 & 900 & 64.7 & 18 \\
\hline$[\mathrm{N}$ II $] 6548 \ldots \ldots \ldots .$. & 17 & & & 18 & 95 & 560 & & \\
\hline$[\mathrm{O}$ I $] 6363 \ldots \ldots \ldots \ldots$ & $\ldots$ & 1.0 & $\ldots$ & $\ldots$ & 1.5 & $\ldots$ & $\ldots$ & $\ldots$ \\
\hline$[\mathrm{O}$ I] $6300 \ldots \ldots \ldots \ldots$ & $\ldots$ & 3.0 & $\ldots$ & $\ldots$ & 3.8 & $\ldots$ & $\ldots$ & $\ldots$ \\
\hline He I $5876 \ldots \ldots \ldots \ldots$ & & & 1 & 1.2 & 7.6 & 29 & $\ldots$ & $\ldots$ \\
\hline [O III] $5007 \ldots \ldots \ldots \ldots$ & 122 & 172 & Q1 & 4.4 & 46 & 6700 & $\ldots$ & $\ldots$ \\
\hline [O III $] 4959 \ldots \ldots \ldots$. & 44 & 57 & $\int 81$ & 1.9 & 12 & 1970 & $\ldots$ & $\ldots$ \\
\hline $\mathrm{H} \beta 4861 \ldots \ldots \ldots \ldots$ & 4.7 & 17 & 23 & 2.4 & 38 & 540 & 45 & 11 \\
\hline He II $4686 \ldots \ldots \ldots \ldots$ & 2.5 & 15 & 33 & 1.1: & 20 & 760 & 67: & $6:$ \\
\hline [O II] $3727 \ldots \ldots \ldots$ & $\ldots$ & $\ldots$ & 16 & $\ldots$ & 28 & $\ldots$ & $\ldots$ & $\ldots$ \\
\hline
\end{tabular}

Weaker Emission Features (units: $10^{-15} \mathrm{ergs} \mathrm{s}^{-1} \mathrm{~cm}^{-2}$ )

\begin{tabular}{|c|c|c|c|c|c|c|c|c|}
\hline$[\mathrm{Fe}$ VII] $] 3589 \quad \ldots \ldots \ldots$ & $\ldots$ & $\ldots$ & 18: & $\ldots$ & $\ldots$ & $\ldots$ & $\ldots$ & $\ldots$ \\
\hline \multicolumn{9}{|l|}{ Balmer cont.? } \\
\hline $3650 \ldots \ldots$ & 1.4 & $\ldots$ & $\ldots$ & $\ldots$ & $17:(30)$ & $\ldots$ & $\ldots$ & $\ldots$ \\
\hline C II 4070. & $\ldots$ & $\ldots$ & $\ldots$ & $\ldots$ & 8 & $\ldots$ & $\ldots$ & $\ldots$ \\
\hline C II $4270 \ldots \ldots \ldots \ldots$ & $\ldots$ & $\ldots$ & $\ldots$ & $\ldots$ & 8 & $\ldots$ & $\ldots$ & $\ldots$ \\
\hline $\mathrm{C}$ II $+[\mathrm{O}$ III $] 4370 \ldots$ & $\ldots$ & 7 & 8 & $\ldots$ & 10 & 106 & $\ldots$ & $\ldots$ \\
\hline$C_{\text {II }} 4400 \ldots \ldots \ldots \ldots$ & $\ldots$ & $\ldots$ & $\ldots$ & $\ldots$ & 2 & $\ldots$ & $\ldots$ & $\ldots$ \\
\hline He I $4460 \ldots \ldots \ldots \ldots$ & $\ldots$ & & $\ldots$ & & 3: & $\ldots$ & $\ldots$ & $\ldots$ \\
\hline $\mathrm{O}$ II $+\mathrm{N}$ II $4640 \ldots \ldots$ & $3.7(50)$ & 13 & $11(30)$ & 1.6 & 28 & 430 & 52 & $\ldots$ \\
\hline $4920 \ldots \ldots \ldots \ldots \ldots$ & $\ldots$ & $\ldots$ & $\ldots$ & $\ldots$ & $\ldots$ & $\ldots$ & $\ldots$ & $\mathrm{P}$ \\
\hline $\mathrm{N}$ iा $5010 \ldots \ldots \ldots \ldots$ & $\ldots$ & $\cdots$ & $\cdots$ & $\ldots$ & $\ldots$ & $\cdots$ & $\cdots$ & $\mathrm{P}$ \\
\hline $\mathrm{N}$ II $+\left[\mathrm{N}_{\mathrm{I}}\right] 5190 \ldots \ldots$ & $\ldots$ & $\ldots$ & $\ldots$ & $\ldots$ & $\mathrm{P}$ & $\ldots$ & $\ldots$ & $\ldots$ \\
\hline $5390 \quad \ldots \ldots \ldots \ldots \ldots$ & $\ldots$ & $\ldots$ & $\ldots$ & $\ldots$ & $\ldots$ & P: & $\ldots$ & $\ldots$ \\
\hline He II $5410 \quad \ldots \ldots \ldots$ & $\ldots$ & $\ldots$ & $\mathrm{P}$ & $\ldots$ & $\ldots$ & P: & $\ldots$ & $\ldots$ \\
\hline$\left[\mathrm{O}_{\mathrm{I}}\right] 5570 \ldots \ldots \ldots \ldots$ & $\ldots$ & $\ldots$ & $\mathrm{P}(80)$ & $\ldots$ & P: & $\cdots$ & $\ldots$ & $\cdots$ \\
\hline $\mathrm{N}$ II $5670 \ldots \ldots \ldots \ldots$ & $\ldots$ & $\ldots$ & $\ldots$ & $\ldots$ & $\mathrm{P}$ & $\ldots$ & $\ldots$ & $\ldots$ \\
\hline$[\mathrm{N}$ iI $] 5760 \ldots \ldots \ldots$ & $\ldots$ & $\ldots$ & $\ldots$ & $\ldots$ & $\mathbf{P}$ & $\mathbf{P}$ & $\ldots$ & $\ldots$ \\
\hline C IV? $5810 \ldots \ldots \ldots$ & $\ldots$ & $\ldots$ & 1 & $\ldots$ & $\mathrm{P}$ & $\ldots$ & $6(20)$ & $\ldots$ \\
\hline $7160 \ldots \ldots \ldots \ldots \ldots$ & $\ldots$ & $1.3:(50)$ & $\ldots$ & $\ldots$ & $\ldots$ & 21 & $\ldots$ & $\ldots$ \\
\hline C II $7230 \ldots \ldots \ldots \ldots$ & $\ldots$ & 2.1 & $\ldots$ & $\ldots$ & $2(20)$ & 11 & $\ldots$ & $\ldots$ \\
\hline $\mathrm{N}$ i? $8210 \ldots \ldots \ldots \ldots$ & $\ldots$ & $9(20)$ & $\ldots$ & $\ldots$ & $6(20)$ & 22: & $\ldots$ & $\ldots$ \\
\hline $\mathrm{N}$ i? 8240 & $\ldots$ & .. & $3(30)$ & $\ldots$ & $\ldots$ & $\ldots$ & $\ldots$ & $\ldots$ \\
\hline \multicolumn{9}{|c|}{ Continuum at $6000 \AA$ (units: $10^{-15} \mathrm{ergs} \mathrm{s}^{-1} \mathrm{~cm}^{-2} \AA^{-1}$ ) } \\
\hline$\ldots$ & 0.03 & 0.57 & 0.28 & 0.03 & 0.81 & 27 & 3.0 & 0.25 \\
\hline
\end{tabular}

from infrared photometry lie far below the mean line. No acceptable increase in $A_{V}^{\prime}$ can be made to put them on the mean relationship. In the case of Nova Cyg 1975 the infrared analysis refers to the initial 3 days after maximum, i.e. to a pseudophotosphere, rather than to a subsequently formed dust shell, as is the case for NQ Vul 1976 and for Nova Ser 1978. Apparently in both these latter novae, as suggested by Szkody et al. (1979), the dust shell is optically thin ${ }^{3}$ (as is also the case for the dust shell around Nova Cyg 1978 observed by Gehrz et al. $1980 \mathrm{~b}$ ). The deduced absolute magnitudes for these

${ }^{3}$ Both Szkody et al. 1979 and Gehrz et al. $1980 a$ used 1240 $\mathrm{km} \mathrm{s}^{-1}$ (from Bappu and Prabhu 1978) as $v$ (exp). This is a factor of 2 larger than our $v$ (exp), which is from a well-exposed, high signal-to-noise ratio, relatively high dispersion $\mathrm{CCD}$ spectrum and must be correct. 
TABLE 5

Shell MASSES (Hydrogen only) ${ }^{\mathrm{a}}$

\begin{tabular}{|c|c|c|}
\hline Object & $n_{e}\left(\mathrm{~cm}^{-3}\right)$ & $M_{\mathrm{sh}}\left(M_{\odot}\right)$ \\
\hline V1500 Cyg 1975 & $1.1 \times 10^{4}$ & $1.9 \times 10^{-5}$ \\
\hline FH Ser $1970 \ldots$. & $1.6 \times 10^{4}$ & $2.0 \times 10^{-5}$ \\
\hline HR Del $1967 .$. & $1.4 \times 10^{4}$ & $2.2 \times 10^{-5}$ \\
\hline V533 Her $1963 \ldots$ & $4.3 \times 10^{3}$ & $2.0 \times 10^{-5}$ \\
\hline Cyg $1920 \ldots \ldots . . . .$. & $3.7 \times 10^{2}$ & $1.3 \times 10^{-4}$ \\
\hline
\end{tabular}

${ }^{\text {a Assumes }} a=b=0.1$ (see text).

novae are rejected. (They are thus also not included in Table 4.) Figure 5, excluding these two points and HR Del, gives via a least squares fit,

$$
M_{V}(\max )=-10.42 \pm 0.38+(1.76 \pm 0.31) \log \left(t_{2}\right)
$$

with a correlation coefficient $\left(r^{2}\right)$ of 0.76 . This will serve as the preliminary galactic calibration for determining the distances to extragalactic novae in subsequent papers and is independent of the controversial distance to the Hyades cluster.

\section{SUMMARY}

A search to find more spatially resolved nova shells has resulted in the discovery of shells around FH Ser 1970 and V533 Her 1963. New angular size measurements for older shells imply they are coasting out at constant velocity. Spectrophotometric scans of the newly discovered objects and others previously known has led to new expansion velocities, measurements of interstel-

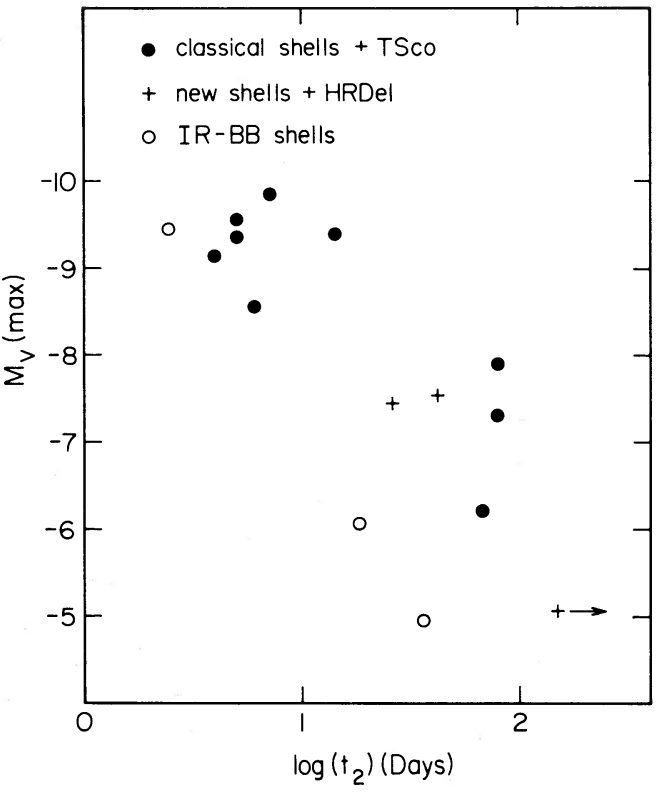

FIG. 5.-The absolute visual magnitude at maximum light-rate of decline relationship for galactic novae is shown. The nova shells discovered in the past 2 years are indicated by crosses, the previously known shells (plus T Sco in M80) by solid circles, and the three novae with infrared-blackbody angular diameters are shown as open circles.

lar absorption, and determinations of the ejected mass. These data are used to derive a new calibration of the absolute visual magnitude at maximum light-rate of decline relationship for galactic novae.

\section{REFERENCES}

Bappu, M. K. V., and Prabhu, T. P. 1978, IAU Circ., No. 3201

Barnes, T. G., and Evans, N. R. 1970, Pub. A.S.P., 82, 889.

Blanco, V. M., Demers, S., Douglass, G. G., and Fitzgerald, M. P. 1970, Pub. US Naval Obs., Series II, 21.

Borra, E. F., and Andersen, P. H. 1970, Pub. A.S.P., 82, 1070.

Chincarini, G. 1964, Astr. Ap., 76, 289.

Cohen, J. G. 1975, Ap. J., 197, 117.

Gallagher, J. S., and Code, A. D. 1974, Ap. J., 189, 303

Gallagher, J. S., Hege, E. K., Kopriva, D. A., Williams, R. E., and Butcher, H. R., 1980, Ap. J., $237,55$.

Gallagher, J. S., and Ney, E. P. 1976, Ap. J. (Letters), 204, L35.

Gehrz, R. D., Grasdalen, G. L., Hackwell, J. A., and Ney, E. P. 1980 a, Ap. J., 237, 855.

Gehrz, R. D., Hackwell, J. A., Grasdalen, G. L., Ney, E. P., Neugebauer, G., and Sellgren, K. 1980b, Ap. J., 239, 570

Harris, W. E., and Racine, R. 1979, Ann. Rev. Astr. Ap., 27, 241.

Hogg, H. S. 1973, in IAU Colloquium 21, Variable Stars in Globular Clusters and in Related Systems, ed. J. D. Fernie (Dordrecht: Reidel), p. 3 .

Humason, M. L. 1938, $A$ p. $J ., 88,228$ 1943, Astr. Ap., 55, 74

Kent, S. M. 1979, Pub. A.S.P., 91, 394

Klemola, A. R. 1968, Pub. A.S.P., 80, 706.

Kohoutek, L. 1981, M.N.R.A.S., 196, 87P

McLaughlin, D. B. 1960, in Stars and Stellar Systems, Vol. 6, Stellar Atmospheres, ed. J. L. Greenstein (Chicago: University of Chicago Press), p. 585.
Ney, E. P., and Hatfield, B. F. 1978, Ap. J. (Letters), 219, L111. Oke, J. B., and Gunn, J. E. $1982 a$, Pub. A.S.P., 94, 586. 1982 b, Pub. A.S.P., in press.

Payne-Gaposchkin, C. 1957, The Galactic Novae (New York: Dover), pp. 183 and 190.

Robbins, R. R., and Sanyal, A. 1978, Ap. J., 219, 985

Shectman, S. A., and Hiltner, W. A. 1976, Pub. A.S.P., 88, 960.

Szkody, P., Dyck, H. M., Capps, R. W., Becklin, E. E., and Cruikshank, D. P. 1979, A.J., 84, 1359.

van den Bergh, S. 1976, in Stars and Stellar Systems, Vol 9 , Galaxies and the Universe, ed. A. Sandage, M. Sandage, and J. Kristian (Chicago: University of Chicago Press), p. 509.

Williams, R. E. 1977, in IAU Colloquium 42, The Interaction of Variable Stars with their Environment, ed. R. Kippenhahn, J. Rahe, and W. Strohmeier, Veröff. Remeis-Sternw. Bamberg, Bd. 11, No. 121 , p. 242. 1982, Ap. J. (Letters), 261, L77.

Williams, R. E., and Gallagher, J. S., 1979, Ap. J., 228, 482.

Williams, R. E., Woolf, N. J., Hege, E. K., Moore, R. E., and Kopriva, D. A. 1978, Ap. J., 224, 171.

Young, P. J., Corwin, H. G., Jr., Bryan, J., and de Vaucouleurs, G., 1976, Ap. J., 209, 882.

Judith G. Cohen and ANN J. Rosenthal: 105-24 California Institute of Technology, Pasadena, CA 91125 